\title{
Rectifiability and Lipschitz extensions into the Heisenberg group
}

\author{
Zoltán M. Balogh · Katrin S. Fässler
}

Received: 17 April 2008 / Accepted: 29 August 2008 / Published online: 25 October 2008

C Springer-Verlag 2008

\begin{abstract}
Denote by $\mathbb{H}^{n}$ the $2 n+1$ dimensional Heisenberg group. We show that the pairs $\left(\mathbb{R}^{k}, \mathbb{H}^{n}\right)$ and $\left(\mathbb{H}^{k}, \mathbb{H}^{n}\right)$ do not have the Lipschitz extension property for $k>n$.
\end{abstract}

Keywords Lipschitz extensions · Heisenberg group

Mathematics Subject Classification (2000) $\quad 53 \mathrm{C} 17 \cdot 22 \mathrm{E} 30$

\section{Introduction}

Over the past decades, it has been a subject of incessant research interest to identify the pairs of metric spaces $(X, Y)$ which exhibit the Lipschitz extension property, that is, the pairs for which there is a constant $C>0$ such that every $L$-Lipschitz map $f: A \rightarrow Y$ from an arbitrary subset $A$ of $X$ can be extended to a $C L$-Lipschitz map $F: X \rightarrow Y$. Most classical results in this direction are concerned with target spaces $Y$ having a linear structure, such as Hilbert spaces or Banach spaces (see [7,14,17,22] and the references therein). Fewer results are known about the extension of Lipschitz maps with nonlinear target spaces even though this question becomes relevant in the theory of rectifiability in general metric spaces $[2,10]$. There is, however, a criterion due to Lang and Schlichenmaier (see [16]) which characterizes the Lipschitz extension property of the pair $(X, Y)$, where $X=\mathbb{R}^{n}$ is a Euclidean space and $Y$ is a complete metric space, in terms of a spherical extension property. According to this result it is enough to check whether every Lipschitz map defined on a sphere of dimension

The authors were supported by Swiss National Science Foundation, European Research Council Project GALA and European Science Foundation Project HCAA.

Z. M. Balogh $(\varangle) \cdot$ K. S. Fässler

Department of Mathematics, University of Bern, Sidlerstrasse 5, 3012 Bern, Switzerland e-mail: zoltan.balogh@math.unibe.ch

K. S. Fässler

e-mail: katrin.faessler@math.unibe.ch 
less than $n$ can be extended to a Lipschitz map on the respective ball with a linear control on the Lipschitz constant.

To be more precise, a metric space $Y$ is called Lipschitz $n$-connected if there exists a constant $C>0$ such that for every $m \in\{0, \ldots, n\}$, every $L$-Lipschitz map $f: S^{m} \subset$ $\mathbb{R}^{m+1} \rightarrow Y$ admits a $C L$-Lipschitz extension $F: \overline{B^{m+1}} \subset \mathbb{R}^{m+1} \rightarrow Y$. In [16], it is shown that the pair $\left(\mathbb{R}^{n}, Y\right)$, where $Y$ is a complete metric space, has the Lipschitz extension property if and only if $Y$ is Lipschitz $(n-1)$-connected. More generally, they prove that for an arbitrary metric space $X$ with Nagata dimension (see [3]) less than or equal to $n$ and a Lipschitz $(n-1)$ -connected space $Y$, the pair $(X, Y)$ has the Lipschitz extension property.

In this paper, we study whether the pairs $\left(\mathbb{R}^{k}, \mathbb{H}^{n}\right)$ and $\left(\mathbb{H}^{k}, \mathbb{H}^{n}\right)$, where $\mathbb{H}^{n}$ denotes the $n$th Heisenberg group, have the Lipschitz extension property. It turns out that the answer depends on the values of $k$ and $n$. Let us recall that the Lipschitz extension property holds for the pair $\left(\mathbb{R}, \mathbb{H}^{n}\right)$, for all $n \geq 1$, since $\mathbb{H}^{n}$ is a geodesic space (see $\left.[9,16]\right)$. More generally, for any complete and quasiconvex space $Y$, the pair $(\mathbb{R}, Y)$ has the Lipschitz extension property. The situation becomes increasingly more difficult for higher dimensional source spaces, i.e. for $k \geq 2$. The Lipschitz extension property of $\left(\mathbb{R}^{2}, \mathbb{H}^{n}\right)$ for $n \geq 2$ has recently been shown in [9] and [19]. The proof of this result uses the above described characterization of Lipschitz extendability via the spherical extension property as in [16] and a theorem of Allcock [1] proving the quadratic isoperimetric inequality for $\mathbb{H}^{n}, n \geq 2$ (see also the survey in [11], 0.7 and the open question therein). We conjecture that the Lipschitz extension property holds for the pair $\left(\mathbb{R}^{k}, \mathbb{H}^{n}\right)$ whenever $k \leq n$.

The main result of this note is the counterpart of the above conjecture for $k>n$ :

Theorem 1 Let $k>n$. Then the pairs $\left(\mathbb{R}^{k}, \mathbb{H}^{n}\right)$ and $\left(\mathbb{H}^{k}, \mathbb{H}^{n}\right)$ do not have the Lipschitz extension property.

In fact, we will show that there is a Lipschitz continuous function $\tilde{f}: S^{n} \subset \mathbb{R}^{n+1} \rightarrow \mathbb{H}^{n}$, which cannot be extended to a Lipschitz map defined on $\overline{B^{n+1}}$. To do so, we shall use the fact that $\mathbb{H}^{n}$ is purely $k$-unrectifiable for $k>n$, i.e. subsets of $\mathbb{R}^{k}$ are mapped under $\mathbb{H}^{n}$ valued Lipschitz functions onto sets with vanishing $k$-dimensional Hausdorff measure. The function $\widetilde{f}: S^{n} \rightarrow \mathbb{H}^{n}$ will be constructed as a lift of a Lagrangian $n$-sphere having the property that any continuous extension to $\overline{B^{n+1}}$ has positive filling volume. This will lead to a contradiction and show the non-extendability of $\tilde{f}$ and thus proves Theorem 1 .

The structure of the paper is as follows. In Sect. 2, we recall notation and background results on Heisenberg groups. In Sect. 3, we discuss the notion of rectifiability in the Heisenberg group. In Sect. 4, we give the proof of Theorem 1. The last section is devoted to final comments and open questions. Motivated by Theorem 1, we introduce and discuss the so called Hölder extension property of Lipschitz mappings.

\section{Notation and background results}

In this section, we recall the differential and metric structure of the Heisenberg group $\mathbb{H}^{n}$. This is a special example in the class of the so-called Carnot groups (see, e.g. [6,11]), defined as follows:

Definition 1 A Carnot group $\mathbb{G}$ is a connected, simply connected Lie group, whose Lie algebra $\mathfrak{g}$ is nilpotent and has a stratification

$$
\mathfrak{g}=V_{1} \oplus \cdots \oplus V_{l},
$$


where $\left[V_{1}, V_{j}\right]=V_{j+1}$ for all $j \in \mathbb{N}$ with $V_{j}=\{0\}$ whenever $j>l$. The positive integer $l$ is called the step of the group.

To define the Lie algebra of the Heisenberg group, we consider $2 n+1$ vectors

$$
\left\{X_{1}, \ldots, X_{n}, Y_{1}, \ldots, Y_{n}, T\right\}
$$

which satisfy the commutation relations

$$
\left[X_{i}, Y_{i}\right]=-\left[Y_{i}, X_{i}\right]=-4 T \text { for } 1 \leq i \leq n
$$

and all other commutators vanish.

The vectors $X_{1}, \ldots, X_{n}, Y_{1}, \ldots, Y_{n}$ span a $2 n$-dimensional space $V_{1}$. This generates the following Lie algebra (where the Lie bracket is defined on the basis elements as described above)

$$
\mathfrak{h}_{n}=V_{1} \oplus V_{2}, \quad \text { where } V_{1}:=\operatorname{span}_{\mathbb{R}}\left\{X_{1}, \ldots, X_{n}, Y_{1}, \ldots, Y_{n}\right\} \quad \text { and } \quad V_{2}:=\operatorname{span}_{\mathbb{R}}\{T\} .
$$

We have $\left[V_{1}, V_{1}\right]=V_{2}$ and $\left[V_{1}, V_{2}\right]=0$. The corresponding Lie group, which can be obtained by the exponential mapping, is called the $n t h$ Heisenberg group. It is an example of a non-commutative step-two Carnot group and can be seen as the space $\mathbb{R}^{2 n+1}$, equipped with the group law

$$
(x, y, t) *\left(x^{\prime}, y^{\prime}, t^{\prime}\right)=\left(x+x^{\prime}, y+y^{\prime}, t+t^{\prime}+2 \sum_{i=1}^{n} x_{i}^{\prime} y_{i}-x_{i} y_{i}^{\prime}\right),
$$

where $x=\left(x_{1}, \ldots, x_{n}\right), x^{\prime}=\left(x_{1}^{\prime}, \ldots, x_{n}^{\prime}\right), \quad y=\left(y_{1}, \ldots, y_{n}\right), y^{\prime}=\left(y_{1}^{\prime}, \ldots, y_{n}^{\prime}\right)$ are points in $\mathbb{R}^{n}$ and $t, t^{\prime} \in \mathbb{R}$. The neutral element of the group is given by $e=(0,0,0)$ and the inverse of $p=(x, y, t)$ is $-p=(-x,-y,-t)$.

We can identify the Lie algebra $\mathfrak{h}_{n}$ with the tangent space $T_{e} \mathbb{H}^{n}$ of $\mathbb{H}^{n}$ at the neutral element $e \in \mathbb{H}^{n}$ and, by left translation, assign to each of the (basis) vectors in the Lie algebra a unique left invariant vector field, which we will denote by the same letter, but with an additional tilde,

$$
\tilde{X}_{i}=\frac{\partial}{\partial x_{i}}+2 y_{i} \frac{\partial}{\partial t}, \quad \widetilde{Y}_{i}=\frac{\partial}{\partial y_{i}}-2 x_{i} \frac{\partial}{\partial t}, \quad \widetilde{T}=\frac{\partial}{\partial t} .
$$

These vector fields satisfy the same commutation relations as the corresponding vectors in $\mathfrak{h}_{n}$, where the Lie bracket is defined by its action on smooth functions as $[\widetilde{X}, \widetilde{Y}] f:=$ $\widetilde{X}(\tilde{Y} f)-\widetilde{Y}(\widetilde{X} f)$.

We consider the so called horizontal tangent space $H_{p} \mathbb{H}^{n}=\left\{\widetilde{X}(p) \mid X \in V_{1}\right\}$ and observe that $H_{p} \mathbb{H}^{n}=\operatorname{ker}(\widetilde{\theta})$, where $\widetilde{\theta}=\mathrm{d} t-2 \sum_{i=1}^{n} y_{i} \mathrm{~d} x_{i}-x_{i} \mathrm{~d} y_{i}$. The tangent spaces $H_{p} \mathbb{H}^{n}$ are collected in the horizontal vector bundle $H \mathbb{H}^{n}$, a sub-bundle of the tangent bundle $T \mathbb{H}^{n}$.

A left invariant sub-Riemannian metric on $\mathbb{H}^{n}$ is next defined by using so-called horizontal curves. These are absolutely continuous curves $\gamma:[a, b] \rightarrow \mathbb{H}^{n}$ with tangents lying almost everywhere in the horizontal bundle, i.e. $\gamma^{\prime}(t) \in H_{\gamma(t)} \mathbb{H}^{n}$ for a.e. $t \in[0,1]$. Equivalently, $\gamma$ is horizontal if it satisfies the condition

$$
\gamma_{2 n+1}^{\prime}(t)=2 \sum_{i=1}^{n} \gamma_{i}^{\prime}(t) \gamma_{n+i}(t)-\gamma_{i}(t) \gamma_{n+i}^{\prime}(t) \text { for a.e. } t \in[a, b] .
$$

We define the length of a horizontal curve by using an inner product on $H \mathbb{H}^{n}$ for which the vectors $\widetilde{X}_{1}(p), \ldots, \widetilde{X}_{n}(p), \widetilde{Y}_{1}(p), \ldots, \widetilde{Y}_{n}(p)$ form an orthonormal basis of $H_{p} \mathbb{H}^{n}$ at each 
$p \in \mathbb{H}^{n}$. More precisely,

$$
\operatorname{length}_{h}(\gamma):=\int_{a}^{b}\left(\left\langle\gamma^{\prime}(t), \gamma^{\prime}(t)\right\rangle_{\gamma(t)}\right)^{\frac{1}{2}} \mathrm{~d} t=\int_{a}^{b}\left(\sum_{i=1}^{2 n} \gamma_{i}^{\prime}(t)^{2}\right)^{\frac{1}{2}} \mathrm{~d} t .
$$

Definition 2 The Carnot-Carathéodory metric is defined as

$$
d_{c c}(f(p), f(q)):=\inf \text { length }_{h}(\gamma),
$$

where the infimum is taken over all horizontal curves $\gamma$ joining $p$ to $q$.

Notice that the existence of the horizontal curves connecting an arbitrary pair of points in the above definition is guaranteed by Chow's theorem (see [11]).

The topology induced by the Carnot-Carathéodory metric coincides with the usual Euclidean topology on the underlying space. However, the $d_{c c}$-metric on $\mathbb{H}^{n}$ is not bi-Lipschitz equivalent to the Euclidean metric $d_{E}$ on $\mathbb{R}^{2 n+1}$. But there is the following comparison of the two metrics: Locally, one can find a constant $C>0$ such that

$$
\frac{1}{C} d_{E}(p, q) \leq d_{c c}(p, q) \leq C d_{E}(p, q)^{\frac{1}{2}} \quad p, q \in B,
$$

where $B$ is a bounded subset in $\mathbb{H}^{n}$ and the constant $C$ depends on the bound of $B$ (see $[4,11])$. This implies that the identity map id $:\left(\mathbb{H}^{n}, d_{c c}\right) \rightarrow\left(\mathbb{R}^{2 n+1}, d_{E}\right)$ is locally Lipschitz. Moreover, it follows directly from the definition that the projection map $\pi:\left(\mathbb{H}^{n}, d_{c c}\right) \rightarrow$ $\left(\mathbb{R}^{2 n}, d_{E}\right)$ is 1 -Lipschitz continuous, indeed

$$
d_{E}(\pi(p), \pi(q)) \leq \text { length }(\pi(\gamma))=\operatorname{length}_{h}(\gamma)=d_{c c}(p, q) \quad p, q \in \mathbb{H}^{n},
$$

where $\gamma$ is a horizontal curve of minimal length which joins $p$ to $q$.

For an arbitrary metric space $(X, d)$ and $0 \leq s<\infty$, one can define the $s$-dimensional Hausdorff measure $\mathscr{H}_{(X, d)}^{s}$ (see, e.g. [20]). We will omit the subscript and simply write $\mathscr{H}^{s}$ if it is clear with respect to which metric the Hausdorff measure should be taken. Also, we abbreviate $\mathscr{H}_{\left(\mathbb{R}^{n}, d_{E}\right)}^{s}=\mathscr{H}_{E}^{s}$ and $\mathscr{H}_{\left(\mathbb{H}^{n}, d_{c c}\right)}^{s}=\mathscr{H}_{c c}^{s}$.

Note that the estimate (1) implies

$$
\frac{1}{C} \mathscr{H}_{E}^{s}(A) \leq \mathscr{H}_{c c}^{s}(A) \leq C \mathscr{H}_{E}^{\frac{s}{2}}(A)
$$

for a bounded set $A$ in $\mathbb{R}^{2 n+1}$, where $C$ depends on the bound of $A$. However, this is only a very rough estimate between the Hausdorff measures of sets with respect to the Euclidean and the Carnot-Carathéodory metrics. For example an open set $A$ in $\mathbb{H}^{n}$ is $2 n+1$ dimensional in the Euclidean metric whereas its Hausdorff dimension with respect to $d_{c c}$ is $2 n+2$. For a complete description of the discrepancy between the Hausdorff measures $\mathscr{H}_{E}^{s}$ and $\mathscr{H}_{c c}^{s}$ we refer to [4-6].

\section{Rectifiability in the Heisenberg group}

The notion of rectifiability can be defined in arbitrary metric spaces. Let us recall from [2] the following

Definition 3 A Borel set $S$ in a metric space $(X, d)$ is said to be countably $\mathscr{H}^{k}$-rectifiable if it can be covered-up to a set with $\mathscr{H}^{k}$-measure zero-by a countable family of Lipschitz 
images of subsets of $\mathbb{R}^{k}$, that is, there exists a countable sequence of Lipschitz functions $f_{j}: A_{j} \subset \mathbb{R}^{k} \rightarrow X$ such that $\mathscr{H}_{(X, d)}^{k}\left(S \backslash \bigcup_{j} f_{j}\left(A_{j}\right)\right)=0$.

In the same paper, Ambrosio and Kirchheim observed that the above notion of rectifiability is not suitable for all metric spaces in the sense that a given metric space can be purely $k$-unrectifiable for some $k \in \mathbb{N}$. Let us recall:

Definition 4 A metric space $(X, d)$ is called purely $k$-unrectifiable if $\mathscr{H}^{k}(f(A))=0$ for any Lipschitz map $f: A \rightarrow X$ with $A \subset \mathbb{R}^{k}$.

Ambrosio and Kirchheim have shown in [2] that the first Heisenberg group is purely $k$-unrectifiable for $k=2,3,4$. More generally, $\mathbb{H}^{n}$ is purely $k$-unrectifiable for $k>n$. This result seems to be part of the mathematical folklore for the specialists working in the geometric measure theory of Carnot groups. It follows indeed from the algebraic characterization of purely $k$-unrectifiability in general Carnot groups due to Magnani [18]. Since we were not able to locate an explicit reference for the application of this criterion to $\mathbb{H}^{n}$ for $n>1$, we give the proof of this statement in Proposition 1 for the convenience of the reader.

Let us first recall

Theorem 2 ([18]) Let $\mathbb{G}$ be a Carnot group with Lie algebra $\mathfrak{g}=V_{1} \oplus V_{2} \oplus \cdots \oplus V_{l}$. Then $\mathbb{G}$ is purely $k$-unrectifiable if and only if the first layer $V_{1}$ does not contain any $k$-dimensional Lie subalgebra.

Recall that a Lie subalgebra of $\mathfrak{g}$ is a subspace $\mathfrak{a}$ of $\mathfrak{g}$ which is closed under the Lie bracket, i.e. $[x, y] \in \mathfrak{a}$ for all $x, y \in \mathfrak{a}$.

We will show the following proposition which enables us to apply Theorem 2 in order to prove the $k$-unrectifiability of $\mathbb{H}^{n}$ for $k>n$.

Proposition 1 The first layer $V_{1}$ of the Lie algebra $\mathfrak{h}_{n}=V_{1} \oplus V_{2}$ does not contain any $k$-dimensional Lie subalgebra for $k>n$.

Theorem 3 The nth Heisenberg group $\mathbb{H}^{n}$ is purely $k$-unrectifiable for $k>n$.

Proof of Theorem 3 This follows from Proposition 1 and Theorem 2.

This result suggests that the Euclidean notion of rectifiability as defined above is not suitable for the geometry of the Heisenberg groups, at least not for $k>n$. See the comments in the last section of this note and the references therein for a further discussion on this subject.

Proof of Proposition 1 Let $V$ be a $k$-dimensional subspace of $V_{1}$ with $k>n$. We prove that there must exist two elements $X$ and $Y$ in $V$ such that their Lie bracket $[X, Y]$ is no longer contained in $V_{1}$ and, in particular, it does not belong to $V$. This shows that $V$ cannot be a Lie subalgebra of $\mathfrak{g}$.

The goal is to show that-if the dimension of $V$ is big enough — the space must contain an element $X$ for which also $J X$ belongs to $V$, where $J$ is the complex multiplication with respect to the basis $\left\{X_{1}, \ldots, X_{n}, Y_{1}, \ldots, Y_{n}\right\}$ given by

$$
J=\left(\begin{array}{cc}
0 & E_{n} \\
-E_{n} & 0
\end{array}\right)
$$


and $E_{n}$ denotes the $n$-dimensional identity matrix. We will then show that $[J X, X]$ is a constant multiple of $T$ and hence not contained in $V_{1}$. To see this, notice first that the dimension formula for the injective map $J: V \rightarrow V_{1}$ yields

$$
\operatorname{dim} J V=\operatorname{dim} V=k \geq n+1
$$

which, by a simple linear algebra argument, implies that $V \cap J V \nsupseteq\{0\}$. Thus, there must exist a non-trivial element $X \in V \cap J V$, i.e.

$$
X=\sum_{i=1}^{n} \alpha_{i} X_{i}+\beta_{i} Y_{i} \in V \quad \text { and } \quad J X=\sum_{i=1}^{n} \beta_{i} X_{i}-\alpha_{i} Y_{i} \in V,
$$

where at least one of the coefficients $\alpha_{i}, \beta_{i}$ is non-zero. We compute

$$
[X, J X]=\left[\sum_{i=1}^{n} \alpha_{i} X_{i}+\beta_{i} Y_{i}, \sum_{i=1}^{n} \beta_{i} X_{i}-\alpha_{i} Y_{i}\right]=4 \sum_{i=1}^{n}\left(\alpha_{i}^{2}+\beta_{i}^{2}\right) T=c \cdot T,
$$

where $c \neq 0$. This completes the proof of the proposition.

\section{Proof of the main result}

In this section, we prove the statements of Theorem 1 which are the main results of this paper. We start first by showing that the pair $\left(\mathbb{R}^{k}, \mathbb{H}^{n}\right)$ does not have the Lipschitz extension property for $k>n$. To do so, we define a Lipschitz function $\widetilde{f}: S^{n} \subset \mathbb{R}^{n+1} \rightarrow \mathbb{H}^{n}$ which admits no Lipschitz continuous extension to $\overline{B^{n+1}}$. This map will be obtained as a lift of a Lagrangian map $f: S^{n} \subset \mathbb{R}^{n+1} \rightarrow \mathbb{R}^{2 n}$ for which the image of any continuous extension to $\overline{B^{n+1}}$ must have positive $\mathscr{H}_{E}^{n+1}$ measure. Let us recall the following definition.

Definition 5 A $\mathscr{C}^{1}$-smooth map $f: M^{m} \rightarrow \mathbb{R}^{2 n}$ from an $m$-dimensional manifold $M^{m}$ to the Euclidean space $\mathbb{R}^{2 n}$ equipped with the standard symplectic form $\omega=\sum_{i=1}^{n} \mathrm{~d} x_{i} \wedge \mathrm{d} y_{i}$ is said to be isotropic if $f^{*} \omega=0$ on $M^{m}$. In the case $m=n$, the map is also called Lagrangian.

Proposition 2 For every $n \in \mathbb{N}$, there is a Lagrangian map

$$
f: S^{n} \subset \mathbb{R}^{n+1} \rightarrow \mathbb{R}^{2 n}
$$

such that $\mathscr{H}_{E}^{n+1}\left(F\left(\overline{B^{n+1}}\right)\right)>0$ for every continuous extension

$$
F: \overline{B^{n+1}} \subset \mathbb{R}^{n+1} \rightarrow \mathbb{R}^{2 n} \text { with }\left.F\right|_{S^{n}}=f .
$$

Proof We consider a function $f: S^{n} \rightarrow \mathbb{R}^{2 n}$ which was given in [8] as an example of an exact Lagrangian immersion. This map is defined as follows:

$$
f: S^{n} \subset \mathbb{R}^{n+1} \rightarrow \mathbb{R}^{2 n}, \quad\left(x_{1}, \ldots, x_{n}, x_{n+1}\right) \mapsto\left(x_{1}, x_{1}, \ldots, x_{n} ; x_{1} x_{n+1}, x_{2} x_{n+1}, \ldots, x_{n} x_{n+1}\right),
$$

where $x_{1}^{2}+\cdots+x_{n}^{2}+x_{n+1}^{2}=1$. We leave it to the reader to check that this map is indeed Lagrangian, i.e. $f^{*} \omega=0$ on $S^{n}$, where $\omega$ denotes the standard symplectic form on $\mathbb{R}^{2 n}$. To do so, it is crucial that we only ask the pullback form to vanish on the set $S^{n}$ where the additional constraint $\sum_{i=1}^{n+1} x_{i}^{2}=1$ holds - the trivial extension of $f$ to the ambient would no longer be Lagrangian.

Next, we show that the image of an arbitrary continuous extension $F: \overline{B^{n+1}} \rightarrow \mathbb{R}^{2 n}$ of $f$ has positive $(n+1)$-dimensional (Euclidean) Hausdorff measure. Actually, it suffices to 


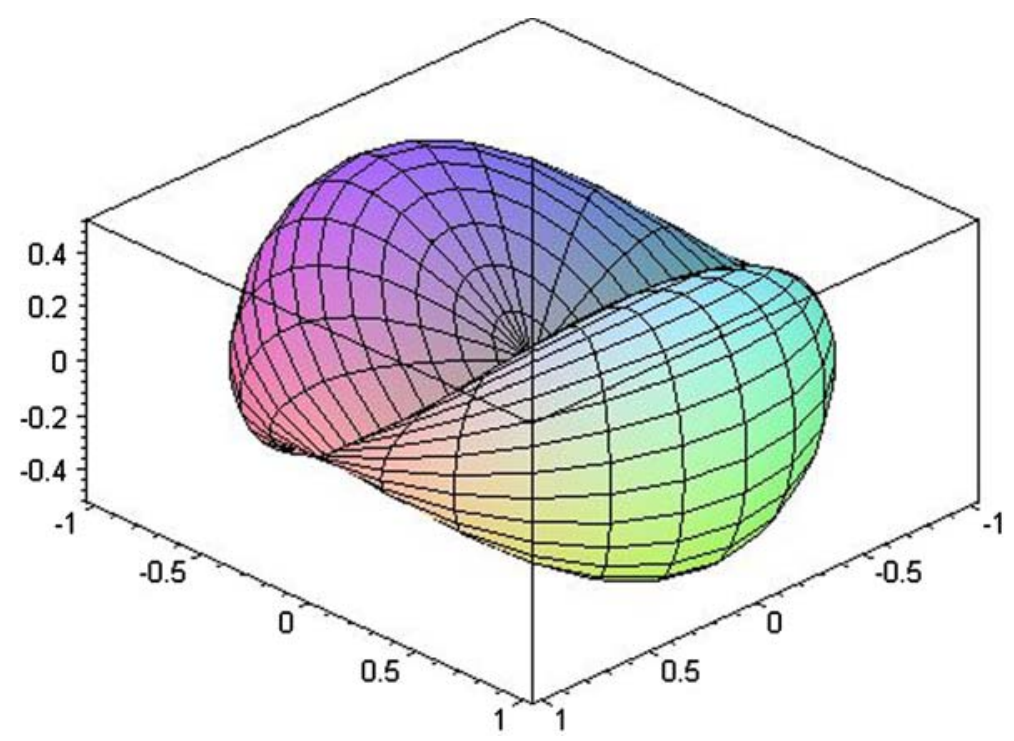

Fig. 1 Knotted sphere-projection of $f\left(S^{2}\right) \subset \mathbb{R}^{4}$ to $\mathbb{R}^{3}$

prove that the projection of the image to an $(n+1)$-dimensional subspace has non-vanishing measure. This follows from the fact that

$$
\pi_{n+1}: \mathbb{R}^{2 n} \rightarrow \mathbb{R}^{n+1}, \quad\left(y_{1}, \ldots, y_{n+1}, \ldots, y_{2 n}\right) \mapsto\left(y_{1}, \ldots, y_{n+1}\right)
$$

is 1-Lipschitz continuous and hence $\mathscr{H}_{E}^{n+1}\left(\pi_{n+1}\left(F\left(\overline{B^{n+1}}\right)\right)\right) \leq \mathscr{H}_{E}^{n+1}\left(F\left(\overline{B^{n+1}}\right)\right)$.

We wish therefore to show that for any continuous extension $F$ of $f$ the composition

$$
\pi_{n+1} \circ F: \overline{B^{n+1}} \rightarrow \mathbb{R}^{n+1}
$$

is onto on a set of positive $(n+1)$-dimensional Hausdorff measure. This will be achieved by using a degree theory (see e.g. [21]) argument as follows.

Let us consider the function

$$
g:=\pi_{n+1} \circ f: S^{n} \rightarrow \mathbb{R}^{n+1}, \quad\left(x_{1}, \ldots, x_{n}, x_{n+1}\right) \mapsto\left(x_{1}, \ldots, x_{n}, x_{1} x_{n+1}\right)
$$

and its trivial extension to $\overline{B^{n+1}}$ given by

$$
G: \overline{B^{n+1}} \rightarrow \mathbb{R}^{n+1}, \quad\left(x_{1}, \ldots, x_{n}, x_{n+1}\right) \mapsto\left(x_{1}, \ldots, x_{n}, x_{1} x_{n+1}\right) .
$$

Consider for example the point $p=\left(\frac{1}{2}, 0, \ldots, 0\right)$ in $\mathbb{R}^{n+1}$. Its preimage is uniquely determined and equals $\left(x_{1}, \ldots, x_{n}, x_{n+1}\right)=\left(\frac{1}{2}, 0, \ldots, 0\right)$, hence $p \notin G\left(\partial \overline{B^{n+1}}\right)=g\left(S^{n}\right)$. Computing the Jacobian of $G$, we find that $J_{G}\left(\frac{1}{2}, 0, \ldots, 0\right)=\frac{1}{2} \neq 0$, which shows that the point $p$ is a regular value of $G$ and its Brouwer degree is non-vanishing, $d\left(G, B^{n+1}, p\right) \neq 0$. Note here, that the map $G$, obtained by projecting $f$ and taking its trivial extension to the interior of $S^{n}$, gives a smooth map from an open subset of $\mathbb{R}^{n+1}$ to $\mathbb{R}^{n+1}$, which ensures that the degree theory is applicable. Since the set $\mathbb{R}^{n+1} \backslash g\left(S^{n}\right)$ is open, there exists $\varepsilon>0$ such that

$$
B(p, \varepsilon) \subset \mathbb{R}^{n+1} \backslash G\left(\partial \overline{B^{n+1}}\right) .
$$


Using the fact that the degree is constant on the connected components of $\mathbb{R}^{n+1} \backslash G\left(\partial \overline{B^{n+1}}\right)$, we find that $d\left(G, B^{n+1}, q\right) \neq 0$ for all $q \in B(p, \varepsilon)$ and thus also $d\left(\pi_{n+1} \circ F, B^{n+1}, q\right) \neq 0$ on $B(p, \varepsilon)$ for an arbitrary continuous extension $F$ of $f$. This is so, because two continuous functions on $\overline{B^{n+1}}$ which coincide on the boundary $\partial \overline{B^{n+1}}=S^{n}$ have the same Brouwer degree relative to $B^{n+1}$ for all points $p$ which do not lie in the image of the boundary. Recall that $d\left(\pi_{n+1} \circ F, B^{n+1}, q\right) \neq 0$ guarantees the existence of a point $x \in B^{n+1}$ such that $\pi_{n+1} \circ F(x)=q$. We conclude that

$$
B(p, \varepsilon) \subseteq \pi_{n+1} \circ F\left(\overline{B^{n+1}}\right),
$$

which yields

$$
0<\mathscr{H}_{E}^{n+1}(B(p, \varepsilon)) \leq \mathscr{H}_{E}^{n+1}\left(\pi_{n+1} \circ F\left(\overline{B^{n+1}}\right)\right) \leq \mathscr{H}_{E}^{n+1}\left(F\left(\overline{B^{n+1}}\right)\right)
$$

and thus completes the proof of the proposition.

We are now in a position to prove Theorem 1:

Proof of Theorem 1 We start with the first statement of the theorem, i.e. we show that the pair $\left(\mathbb{R}^{k}, \mathbb{H}^{n}\right)$ does not have the Lipschitz extension property for $k>n$. It suffices to find an index $m \in\{0, \ldots, k-1\}$ and a Lipschitz function $\widetilde{f}: S^{m} \subset \mathbb{R}^{m+1} \rightarrow \mathbb{H}^{n}$ which admits no Lipschitz extension $\widetilde{F}: \overline{B^{m+1}} \subset \mathbb{R}^{m+1} \rightarrow \mathbb{H}^{n}$. Proposition 2 will be helpful to construct an example of such a function $\tilde{f}$ for $m=n$. The non-existence of the Lipschitz extension for $\widetilde{f}$ will be shown using the unrectifiability result in Theorem 3 .

The desired mapping $\widetilde{f}$ will be the so called Legendrian lift of the Lagrangian mapping $f$ from Proposition 2. To explain the construction of a Legendrian lift consider the 1-form $\theta=2 \sum_{i=1}^{n} y_{i} \mathrm{~d} x_{i}-x_{i} \mathrm{~d} y_{i}$ on $\mathbb{R}^{2 n}$. We note that $\mathrm{d} \theta=-4 \omega$, where $\omega$ denotes the standard symplectic form. One can lift the function $f$ from Proposition 2 to a map $\widetilde{f}=(f, h)$ : $S^{n} \rightarrow \mathbb{R}^{2 n} \times \mathbb{R}=\mathbb{H}^{n}$, where $h$ is defined up to an additive constant uniquely by the relation $f^{*} \theta=\mathrm{d} h$. To see that $h$ is well defined by the above condition, we use the Poincare lemma, the fact that $S^{n}$ is simply connected and the closedness of the one-form $f^{*} \theta$. The last condition follows since $f$ is Lagrangian: $\mathrm{d} f^{*} \theta=f^{*} \mathrm{~d} \theta=-4 f^{*} \omega=0$. Alternatively, $h$ can be obtained by choosing an arbitrary point $x_{0} \in S^{n}$ and setting

$$
h(x):=\int_{f \circ \alpha} \theta \quad x \in S^{n}
$$

where $\alpha:[0,1] \rightarrow S^{n}$ is a smooth curve in $S^{n}$ which joins $x_{0}$ to $x$. One can apply Stokes' theorem and the fact that $f$ is Lagrangian on the simply connected manifold $S^{n}$ in order to see that $h$ is well-defined by the above formula, that is, the integral does not depend on the choice of the curve $\alpha$. We leave it as an exercise for the interested reader to verify that for the Lagrangian mapping $f$ from Proposition 2 the function $h$ described as above is given by $h(x)=\frac{2}{3} x_{n+1}^{3}-2 x_{n+1}+c$, where $c \in \mathbb{R}$ is an arbitrary additive constant.

Now observe that the mapping $\tilde{f}: S^{n} \rightarrow \mathbb{H}^{n}$ is indeed Legendrian, i.e. it satisfies the condition $\tilde{f}^{*} \widetilde{\theta}=0$, where $\widetilde{\theta}=\mathrm{d} t-2 \sum_{i=1}^{n} y_{i} \mathrm{~d} x_{i}-x_{i} \mathrm{~d} y_{i}$. It is a well-known fact (see e.g. [11]), that every smooth Legendrian map $\tilde{f}: S^{n} \rightarrow \mathbb{R}^{2 n+1}$ is Lipschitz as a map from $S^{n}$ to $\mathbb{H}^{n}$. In particular, our function $\tilde{f}=(f, h): S^{n} \rightarrow \mathbb{H}^{n}$ is Lipschitz. Projecting this map to $\mathbb{R}^{2 n}$, we obtain the function $f: S^{n} \rightarrow \mathbb{R}^{2 n}$ from Proposition 2 . We know that

$$
\mathscr{H}_{E}^{n+1}\left(F\left(\overline{B^{n+1}}\right)\right)>0
$$

for any continuous extension $F: \overline{B^{n+1}} \rightarrow \mathbb{R}^{2 n}$. 
Now, assume that our function $\tilde{f}: S^{n} \rightarrow \mathbb{H}^{n}$ could be extended to a Lipschitz continuous function $\widetilde{F}: \overline{B^{n+1}} \rightarrow \mathbb{H}^{n}$. Its projection $F: \overline{B^{n+1}} \rightarrow \mathbb{R}^{2 n}$ would then also be Lipschitz continuous and should hence satisfy $\mathscr{H}_{E}^{n+1}\left(F\left(\overline{B^{n+1}}\right)\right)>0$. But then

$$
\mathscr{H}_{c c}^{n+1}\left(\widetilde{F}\left(\overline{B^{n+1}}\right)\right) \geq \mathscr{H}_{E}^{n+1}\left(F\left(\overline{B^{n+1}}\right)\right)>0,
$$

where we have used the 1-Lipschitz continuity of the projection $\pi:\left(\mathbb{H}^{n}, d_{c c}\right) \rightarrow\left(\mathbb{R}^{2 n}, d_{E}\right)$ (see Eq. (2)). Yet, (3) is a contradiction to Theorem 3. We conclude that the function $\tilde{f}$ does not possess any Lipschitz continuous extension $\widetilde{F}$ to $\overline{B^{n+1}}$ and hence $\left(\mathbb{R}^{k}, \mathbb{H}^{n}\right)$ does not have the Lipschitz extension property. This concludes the proof of the first statement of Theorem 1.

We turn now to the second statement, i.e. we shall prove that the pair $\left(\mathbb{H}^{k}, \mathbb{H}^{n}\right)$ does not have the Lipschitz extension property for $k>n$. Let us consider the function $\widetilde{f}: S^{n} \subset$ $\mathbb{R}^{n+1} \rightarrow \mathbb{H}^{n}$ which was given in the proof of the first statement as an example of a Lipschitz map which has no Lipschitz continuous extension to $\overline{B^{n+1}}$. This can also be considered as a map from a subset of $\mathbb{H}^{k}$ to $\mathbb{H}^{n}, k>n$ :

$$
\widetilde{g}: S \subset \mathbb{H}^{k} \rightarrow \mathbb{H}^{n}, \quad \widetilde{g}:(x, y, t) \mapsto \widetilde{g}(x, y, t):=\widetilde{f}\left(x_{1}, \ldots, x_{n+1}\right),
$$

where $S:=\left\{\left(x_{1}, \ldots, x_{n+1}, 0, \ldots, 0\right) \in \mathbb{H}^{k}: x_{1}^{2}+\cdots+x_{n+1}^{2}=1\right\}$. Clearly, this new map is also Lipschitz, since the $d_{c c}$-metric on $\left\{(x, 0,0): x \in \mathbb{R}^{k}\right\} \subset \mathbb{H}^{k}$ is just the usual Euclidean one on $\mathbb{R}^{k}$.

Now, if $\left(\mathbb{H}^{k}, \mathbb{H}^{n}\right)$ had the Lipschitz extension property, $\widetilde{g}$ could be extended to a Lipschitz map $\widetilde{G}: \mathbb{H}^{k} \rightarrow \mathbb{H}^{n}$. But then restricting this to the set $\left\{\left(x_{1}, \ldots, x_{n+1}, 0, \ldots, 0\right)\right.$ : $\left.\left(x_{1}, \ldots, x_{n+1}\right) \in \mathbb{R}^{n+1}\right\} \subset \mathbb{H}^{k}$ would yield a Lipschitz extension $\widetilde{F}: \mathbb{R}^{n+1} \rightarrow \mathbb{H}^{n}$ of the map $\tilde{f}: S^{n} \subset \mathbb{R}^{n+1} \rightarrow \mathbb{H}^{n}$. We have explained in the first part of the proof why such an extension cannot exist. We conclude that $\left(\mathbb{H}^{k}, \mathbb{H}^{n}\right)$ does not have the Lipschitz extension property for $k>n$.

\section{Final comments and questions}

In this section we shall collect a number of remarks and questions related to the Lipschitz extensions of mappings with Heisenberg group as targets.

Remark 1 Let us notice first that our Theorem 1 continues to hold for the Heisenberg group equipped with any metric bi-Lipschitz equivalent to the Carnot-Carathéodory metric. This includes the class of dilation invariant homogenous metrics.

Motivated by the non-existence of the Lipschitz extensions formulated in Theorem 1 we can ask whether extensions exist in the larger class of Hölder continuous functions and what is the best Hölder exponent for which extensions always exists. To state this question let us formulate the following:

Definition 6 The pair of metric spaces $(X, Y)$ satisfies the $\alpha$ (Hölder) extension property if any Lipschitz map $f: A \rightarrow Y$ defined on a subset $A \subset X$ has an $\alpha$-Hölder continuous extension $F: X \rightarrow Y$. We denote the Hölder number of the pair $(X, Y)$ by

$$
\alpha(X, Y)=\sup \{\alpha \leq 1: \text { the pair }(X, Y) \text { has the } \alpha \text { extension property }\} .
$$

Notice that $\alpha(X, Y)=1$ if the pair $(X, Y)$ has the Lipschitz extension property. We would like to pose the problem 
Question 1 Determine the numbers $\alpha\left(\mathbb{R}^{k}, \mathbb{H}^{n}\right)$ and $\alpha\left(\mathbb{H}^{k}, \mathbb{H}^{n}\right)$.

Related to the above question we conjecture that if $k \leq n$ then the pairs $\left(\mathbb{R}^{k}, \mathbb{H}^{n}\right)$ and $\left(\mathbb{H}^{k}, \mathbb{H}^{n}\right)$ have the Lipschitz extension property and so

$$
\text { if } k \leq n \text { then } \alpha\left(\mathbb{R}^{k}, \mathbb{H}^{n}\right)=\alpha\left(\mathbb{H}^{k}, \mathbb{H}^{n}\right)=1 .
$$

Recall that $\alpha\left(\mathbb{R}^{k}, \mathbb{H}^{n}\right)=1$ for $k \leq n$ is equivalent to $\mathbb{H}^{n}$ being Lipschitz $(k-1)$-connected for $k \leq n$. If this is true, we would also obtain that the pair $\left(X, \mathbb{H}^{n}\right)$, where $X$ is an arbitrary metric space with Nagata dimension less than or equal to $n$, had the Lipschitz extension property.

To this date we only know that $\alpha\left(\mathbb{R}^{k}, \mathbb{H}^{n}\right)=1$ for the values $k \leq n, k=1,2$. As mentioned in the introduction, the case $k=2$ follows from the work of Allcock [1] as shown in [9] and independently in [19]. It is at the moment not clear to us how to generalize Allcock's result to higher values of $k$. For the second problem with the Heisenberg group as source space we do not know if the Lipschitz extension property holds even for $k=1$ or $k=2$. It seems that the problem is of different nature than the one with Euclidean space as source and a new technique should be developed.

Related to Question 1 we believe that the situation changes drastically in the case $k>n$. Going beyond the result of Theorem 1 we conjecture that

$$
\text { if } k>n \text { then } \alpha\left(\mathbb{R}^{k}, \mathbb{H}^{n}\right)=\alpha\left(\mathbb{H}^{k}, \mathbb{H}^{n}\right)=1 / 2 \text {. }
$$

This conjecture is motivated by a related conjecture of [11] asking for the best Hölder exponent of homeomorphisms $f: \mathbb{R}^{3} \rightarrow \mathbb{H}^{1}$ where Gromov conjectures the same value $1 / 2$. We mention the recent results of Hajłasz and Tyson [13] who construct onto maps $f: \mathbb{R}^{3} \rightarrow \mathbb{H}^{1}$ that are Hölder continuous with exponent arbitrary close to $3 / 4$. The same authors are using the results of the present paper in their upcoming work [12] to show that Lipschitz mappings are not dense in the space of Sobolev mapping with Heisenberg group targets.

Remark 2 The phenomenon observed in Theorem 3-the purely $k$-unrectifiability of $\mathbb{H}^{n}$ for $k>n$-suggests that the Euclidean notion of rectifiability as given in Definition 4 is not suitable for the geometry of the Heisenberg groups, at least not for $k>n$.

See [15] and [10] for a discussion on this subject and a new, alternative notion of rectifiability which better fits the Heisenberg group. The authors introduce so-called $\mathbb{H}$-regular surfaces and observe very different properties of these objects, depending on their dimension $k$. The $k$-dimensional $\mathbb{H}$-regular surfaces $(1 \leq k \leq n)$ are Euclidean submanifolds with equal topological, metric and Euclidean dimension-horizontal curves for $k=1$, submanifolds of Legendrian manifolds for $k<n$ and Legendrian manifolds for $k=n$. On the other hand, $k$-codimensional $\mathbb{H}$-regular surfaces are in general far from being smooth Euclidean manifolds and their metric dimension exceeds their topological dimension. In fact it follows from the results of $[5,6]$ that not just for regular submanifolds but even for general sets there is a discrepancy between the Hausdorff dimension of sets with respect to $d_{E}$ and $d_{c c}$. An alternative way of proving the failure of a certain extension property in some class of Hölder regularity would be to look at the obstruction given by the discrepancy of Hausdorff dimension of sets in the two metrics.

Acknowledgments We thank Nathan Habegger, Raul Serapioni and Valentino Magnani for helpful communications about the subject of this paper. Figure 1 was produced using MAPLE. 


\section{References}

1. Allcock, D.: An isoperimetric inequality for the Heisenberg groups. Geom. Funct. Anal. 8(2), 219-233 (1998)

2. Ambrosio, L., Kirchheim, B.: Rectifiable sets in metric and Banach spaces. Math. Ann. 318(3), 527-555 (2000)

3. Assouad, P.: Sur la distance de Nagata. C. R. Acad. Sci. Paris Sér. I Math. 294(1), 31-34 (1982)

4. Balogh, Z.M., Rickly, M., Serra Cassano, F.: Comparison of Hausdorff measures with respect to the Euclidean and the Heisenberg metric. Publ. Mat. 47(1), 237-259 (2003)

5. Balogh, Z.M., Tyson, J.T., Warhurst, B.: Gromov's dimension comparison problem on Carnot groups. C. R. Math. Acad. Sci. Paris 346(3-4), 135-138 (2008)

6. Balogh, Z.M., Tyson, J.T., Warhurst, B.: Sub-Riemannian vs. Euclidean dimension comparison and fractal geometry on Carnot groups. Adv. Math. (2008, to appear)

7. Brudnyi, A., Brudnyi, Y.: Metric spaces with linear extensions preserving Lipschitz condition. Am. J. Math. 129(1), 217-314 (2007)

8. Ekholm, T., Etnyre, J., Sullivan, M.: Non-isotopic Legendrian submanifolds in $\mathbb{R}^{2 n+1}$. J. Differential Geom. 71(1), 85-128 (2005)

9. Fässler, K.: Extending Lipschitz maps from Euclidean spaces into Heisenberg groups. Master's thesis, Mathematisches Institut, Universität Bern (2007)

10. Franchi, B., Serapioni, R., Serra Cassano, F.: Regular submanifolds, graphs and area formula in Heisenberg groups. Adv. Math. 211(1), 152-203 (2007)

11. Gromov, M.: Carnot-Carathéodory spaces seen from within. In: Sub-Riemannian geometry, Progr. Math., vol. 144, pp. 79-323. Birkhäuser, Basel (1996)

12. Hajłasz, P., Tyson, J.T.: Density of Lipschitz maps in Sobolev spaces with Heisenberg group target (2008, in preparation)

13. Hajłasz, P., Tyson, J.T.: Lipschitz, Holder and Sobolev surjections from Euclidean cubes (2008, in preparation)

14. Johnson, W.B., Lindenstrauss, J., Schechtman, G.: Extensions of Lipschitz maps into Banach spaces. Israel J. Math. 54(2), 129-138 (1986)

15. Kirchheim, B., Serra Cassano, F.: Rectifiability and parameterization of intrinsic regular surfaces in the Heisenberg group. Ann. Sci. Norm. Super. Pisa Cl. Sci. (5) 3(4), 871-896 (2004)

16. Lang, U., Schlichenmaier, T.: Nagata dimension, quasisymmetric embeddings, and Lipschitz extensions. Int. Math. Res. Not. (58), 3625-3655 (2005)

17. Lee, J.R., Naor, A.: Extending Lipschitz functions via random metric partitions. Invent. Math. 160(1), 59-95 (2005)

18. Magnani, V.: Unrectifiability and rigidity in stratified groups. Arch. Math. (Basel) 83(6), 568-576 (2004)

19. Magnani, V.: Contact equations and Lipschitz extensions (2007). ArXiv:0711.5003v1

20. Mattila, P.: Geometry of sets and measures in Euclidean spaces, Cambridge Studies in Advanced Mathematics, vol. 44. Cambridge University Press, Cambridge (1995)

21. Milnor, J.W.: Topology from the differentiable viewpoint. Princeton Landmarks in Mathematics. Princeton University Press (1997). Based on notes by David W. Weaver; Revised reprint of the 1965 original

22. Wells, J.H., Williams, L.R.: Embeddings and extensions in analysis. Springer-Verlag, New York (1975). Ergebnisse der Mathematik und ihrer Grenzgebiete, Band 84 